\title{
Deep levels in homoepitaxial boron- doped diamond films studied by capacitance and current transient spectroscopies
}

\author{
Pierre Muret ${ }^{*}, 1$, Julien Pernot ${ }^{* *}, 1$, Tokuyuki Teraji ${ }^{2}$ and Toshimichi Ito ${ }^{3}$ \\ ${ }^{1}$ Institut Néel-CNRS \& Université Joseph Fourier, 25 avenue des Martyrs, BP 166, 38042 Grenoble Cedex9, FRANCE \\ ${ }^{2}$ Sensor Materials Center, National Institute for Materials Science, 1-1 Namiki, Tsukuba, Ibaraki 305-0044, JAPAN \\ ${ }^{3}$ Graduate School of Engineering, Osaka University, 2-1 Yamada-oka, Suita, Osaka 565-0871, JAPAN
}

Received zzz, revised zzz, accepted zzz

Published online zzz (Dates will be provided by the publisher.)

PACS: $61.72 . \mathrm{Ff}$, 68.55.Ln, 71.55.Cn, 78.60.Hk, 81.05.Uw

* Corresponding authors: pierre.muret@grenoble.cnrs.fr, Phone: +33 476 887893, Fax: +33 476881191

** julien.pernot@ grenoble.cnrs.fr, Phone: +33 476 887460, Fax: +33 476881191

Deep level transient spectroscopies (DLTS) applied to Schottky junctions made on homoepitaxial boron-doped diamond films show the existence of two traps. A deep acceptor, negatively charged and strongly attractive for holes, $1.57 \mathrm{eV}$ above the valence band edge displays the characteristic features of a complex defect due to interacting centers and impurities, also displaying some evolutions after thermal cycles, possibly due to hydrogen effusion or diffusion. It is tentatively ascribed to associa-

\section{Introduction.}

A growing number of devices is based on boron doped diamond, a large gap semiconductor [1,2]. Like in other semiconductors, residual deep centers may hamper the improvement of device performances. But the electrical activity of defects and unwanted impurities are very scarcely documented [3-5], contrary to photoluminescence and cathodoluminescence due to radiative transitions of carriers. Non-radiative transitions are however an essential mechanism which controls the electrical characteristics of electronic devices through capture and emission of carriers trapped on the deep centres. Then, experimental evidence comes naturally from the transient response of junctions, which are sensitive to the trapping and de-trapping events. Such a study is presented here, addressing more samples and bringing new details than already published in [5]. Section 2 contains the experimental techniques and data while section 3 is devoted to the discussion of results. tion of a boron atom, a vacancy and several hydrogen atoms. A deep donor, $1.13 \mathrm{eV}$ above the valence band edge, able to compensate the boron acceptors, is attributed to a defect correlated with dislocations. It could be due to the positively charged carbon vacancy. These conclusions are drawn from the Fourier transform-DLTS results coupled with isothermal time domain algorithms allowing the discrimination of multiple emission rates with high resolution.
2 Experimental techniques and results

2.1 Diamond growth, contacts fabrication and electrical measurements

Boron-doped homoepitaxial layers were deposited on Ib-type (100) diamond substrates, by microwave plasma assisted chemical vapor deposition (MPCVD), with conditions detailed in references [6,7]. Several Al Schottky contacts were fabricated on the oxidized surface using an electron-beam evaporator. Ohmic contacts were also made on the top surface of the diamond film with a gap of some tens of $\mu \mathrm{m}$ between the Schottky diode periphery and the ohmic contact. The resulting series resistance of each diode was low enough for allowing capacitance measurements at $1 \mathrm{MHz}$, which indicated a net acceptor concentration of $(8 \pm 1) \times 10^{16} \mathrm{~cm}^{-3}$ in the sample under study, which will be labelled A. Other homoepitaxial samples were prepared with different conditions in an other reactor, with a very light boron concentration of $7.5 \times 10^{14} \mathrm{~B} / \mathrm{cm}^{-3}[8,9]$. Ohmic contacts were deposited on the sample B of ref. [9], allow- 
ing current measurements in the bulk, because the sample has been treated in an oxygen plasma in order to remove the conductive surface layer. Fourier-transform deep level transient spectroscopy (DLTS) has been performed on the various samples, either with the capacitance detected mode in Schottky diodes of the sample A, or with photo-induced current transient spectroscopy (PICTS) in the sample B, provided only with ohmic contacts.

\subsection{Fourier transform deep level transient spectroscopy}

The capacitance due to the depletion zone of a junction is sensitive to the redistribution of charge which takes place when deep centres are loosing carriers by reemission either to the valence or conduction band. A transient current also exist because a corresponding flow of carriers is driven by the electric field into the external circuit. This capacitance or current transient can be analysed by Fourier transform, either as a function of temperature which controls the emission rates (DLTS), at some time windows $t_{w}$, or varying the time window (Isothermal Transient Spectroscopy abbreviated in ITS) at several temperatures [10].

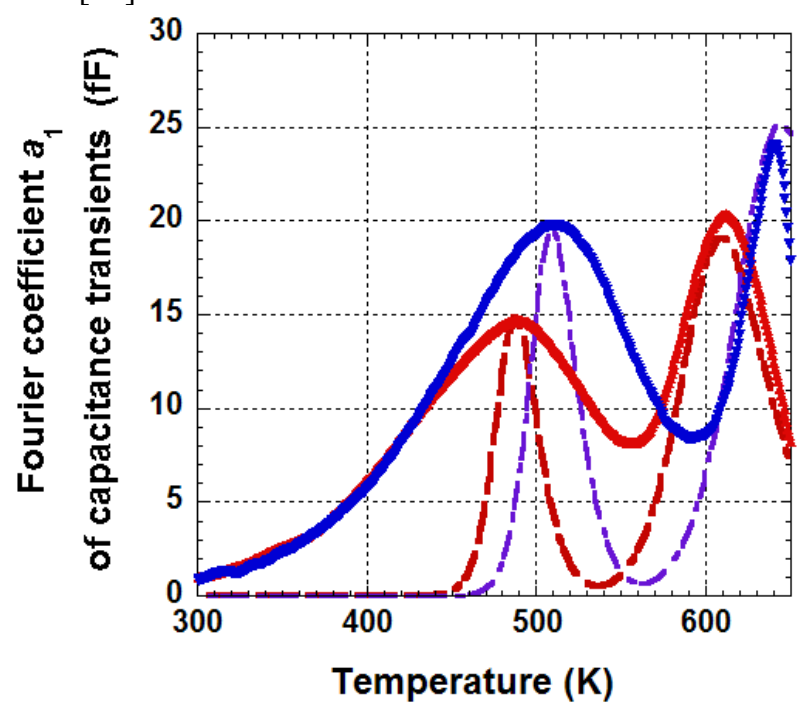

Figure 1 One of the Fourier coefficients, here $a_{1}$, of the experimental capacitance transients in the diode 1 of the sample A, measured in time windows of $2.05 \mathrm{~s}$ and $10 \mathrm{~s}$ respectively for the peaks at the higher and lower temperatures, in full lines. Broken lines simulate the same DLTS spectra, assuming only one emission rate at each temperature, deduced from the Arrhenius diagram in Fig. 3. They deviate markedly from experimental data below $550 \mathrm{~K}$, except for the peak maxima.

The Fourier coefficient $a_{1}$ of the capacitance transients measured in the diode number 1 and 2 of the sample A are displayed respectively in Fig. 1 and 2 for two time windows. They clearly show two peaks, occurring at the same temperatures in the two diodes, but with different amplitudes. This fact indicates that traps are not homogenously scattered in the sample A. The peaks appearing near $500 \mathrm{~K}$ and $630 \mathrm{~K}$ for the two time windows shown in this study will be labelled respectively "trap I" and "trap II". As it can be seen in figures, an other important difference between the two diodes is the width of the peaks related to trap I: in diode 2, the simulated DLTS spectra are very close to the experimental ones, whereas the peak occurring at the lower temperature is much broader in the diode 1 . Such a discrepancy between the experimental and simulated spectra indicates that multi-emission rates probably occur in diode 1 for this trap or alternatively, that several traps superimpose. From the knowledge of the Fourier coefficients amplitude and net acceptor concentration, the trap concentrations are about $3 \times 10^{15} \mathrm{~cm}^{-3}$ in diode 1 for the two traps while in diode 2 , they are close to $10^{16} \mathrm{~cm}^{-3}$ and $10^{15} \mathrm{~cm}^{-3}$ respectively for trap I and II.

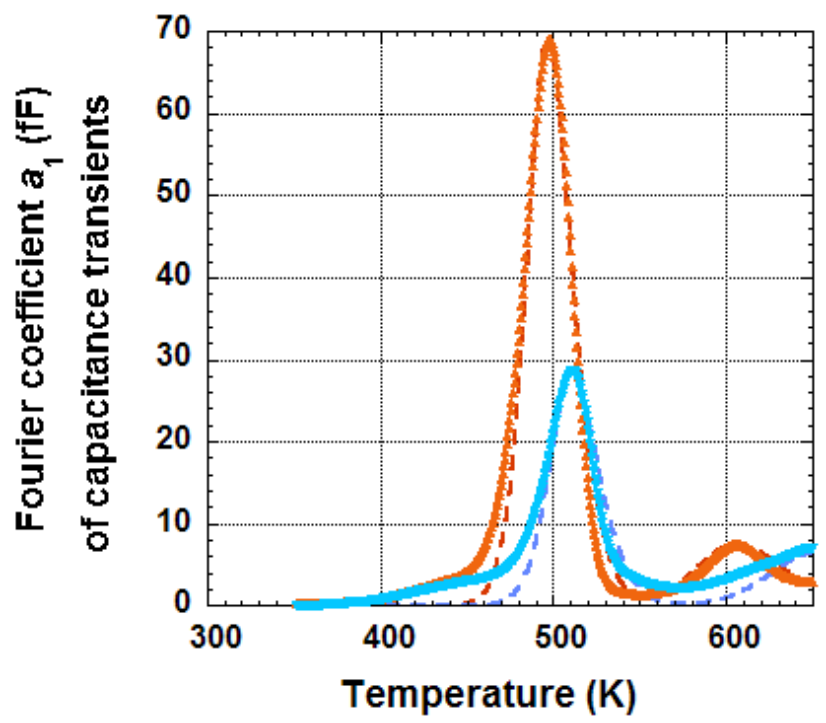

Figure 2 Fourier coefficients $a_{1}$ in diode 2 of sample A for two time windows in full lines: $2.05 \mathrm{~s}$ (the smaller peak) and $10 \mathrm{~s}$ (the taller peak). Although simulated spectra, in broken lines, are very close to experimental curves, the height difference of the peaks near $500 \mathrm{~K}$ (trap I) is not expected for a discrete emission rate.

In a p-type semiconductor, the main purpose of DLTS consists in deriving for each deep level the hole emission rate $e_{p}$, which is thermally activated as

$$
e_{p}=g_{p} \sigma_{p} v_{t h p} N_{V} \exp \left[\left(E_{V}-E_{T}\right) / k T\right]
$$

where $g_{p}$ and $\sigma_{p}$ are respectively the degeneracy factor and capture cross section of the deep level, $v_{t h p}$ is the thermal velocity of holes, $N_{V}$ the effective density of states at the valence band edge, $E_{T}-E_{V}$ the ionisation energy of the deep level, $k$ the Boltzmann constant and $T$ the absolute temperature. As the prefactor contains $T^{2}$ and eventually an exponentially activated factor due to the barrier $\Delta E_{B}$ for capture, $e_{p}$ reads $\alpha T^{2} \exp \left[-E_{a} / k T\right]$ where only the two 
constants $\alpha$ and $E_{a}$ can be extracted from experimental data.

Because the product $e_{p} t_{w}$ has a unique and known value when a particular Fourier coefficient is maximum, numerous data of $e_{p}(T)$ can be collected for each trap. Then, from an Arrhenius plot of $e_{p} / T^{2}$, the activation energy $E_{a}$ and the apparent capture cross section $\sigma_{a p p}$, contained in the $\alpha$ constant, can be deduced respectively from the slope of the straight line and ordinate at infinite temperature. Such a diagram is recognised as the signature of the deep level as long as the experimental points are aligned along a straight line. In fact, the activation energy $E_{a}$ is the sum of $\Delta E_{B}$ and the ionisation enthalpy $\Delta H_{T}$. For the two traps detected in the samples studied here, the Arrhenius diagram is plotted in Fig. 3, with data coming from DLTS spectra obtained in sample A (full symbols) and PICTS data [9] coming from sample B (open symbols).

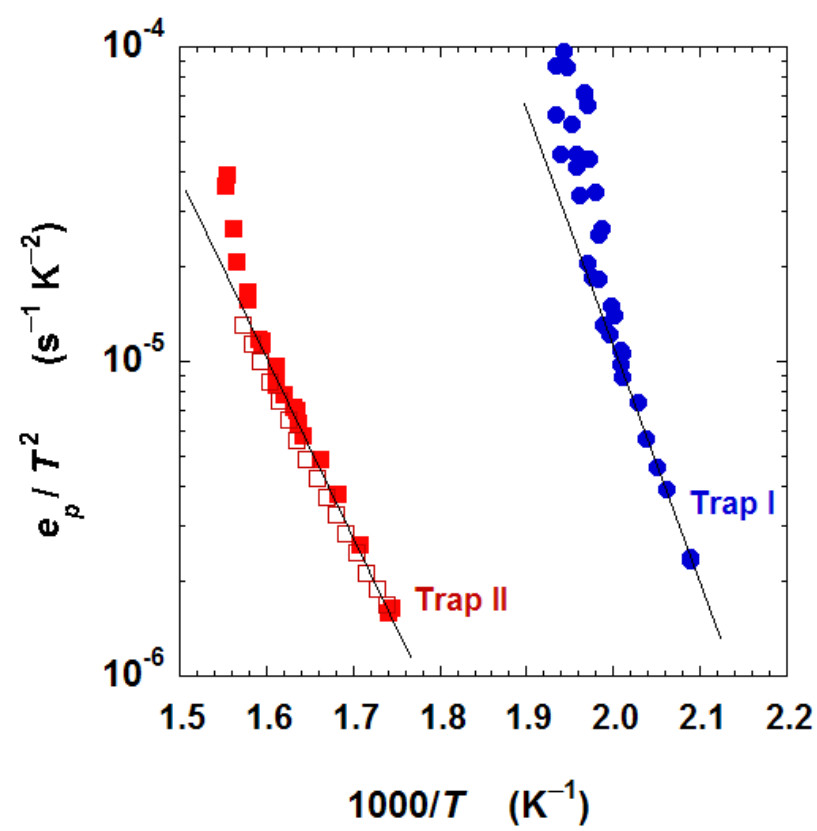

Figure 3 : Arrhenius diagram for the two traps. Full circles and squares come from DLTS spectra in sample A, while open squares from PICTS in sample B. Deviation from straight lines occur at highest temperature (and highest emission rates), specially for trap I.

From the straight lines plotted in Fig. 3, using $40 \%$ of the data for trap I and $80 \%$ for trap II, one deduces $E_{a 1}=1.57 \pm 0.1 \mathrm{eV}, \quad \sigma_{p 1}=(2-8) \times 10^{-12} \mathrm{~cm}^{2}$ for trap I and $E_{a 2}=1.13 \pm 0.06 \mathrm{eV}, \sigma_{p 2}=(1-4) \times 10^{-18} \mathrm{~cm}^{2}$ for trap II.

Although the points located outside the straight lines indicate that the model relying on a unique emission rate at each temperature ceases to be valid above some temperature, the activation energies can be derived from the straight portion with reasonable error bars, typically less than $7 \%$ for the relative uncertainty. As soon as these activation energies are known, the simulated DLTS spectra can be superimposed in Fig. 1 and 2 on the experimental data and the capture cross sections can be adjusted so that maxima match simultaneously those of the experimental curves for both time windows. The capture cross sections derived in such a way are more accurate than those obtained at the intercept of the vertical axis of the Arrhenius diagrams but can still be compared and justify the error bars mentioned above for $\sigma_{p 1}$ and $\sigma_{p 2}$. It is worthwhile to notice that firstly this procedure is not commonly used by DLTS practitioners, and secondly each capture cross section thus obtained is extrapolated at infinite temperature because the capture barrier factor is incorporated into the activation energy. Other possible deviations of the extracted values from the real ones are discussed separately for trap I and II in the following.

\subsection{Properties of the trap I}

The DLTS spectra related to trap I show at least two anomalous behaviours: (i) a large broadening in some diodes ; (ii) a peak height which depends on the time window. These features are not expected if the emission rate distribution is discrete, with a value given by equation (1), or equivalently if the transient does not follow the simple exponential law $\exp \left(-e_{p} t\right)$ at each temperature, $t$ being the time.

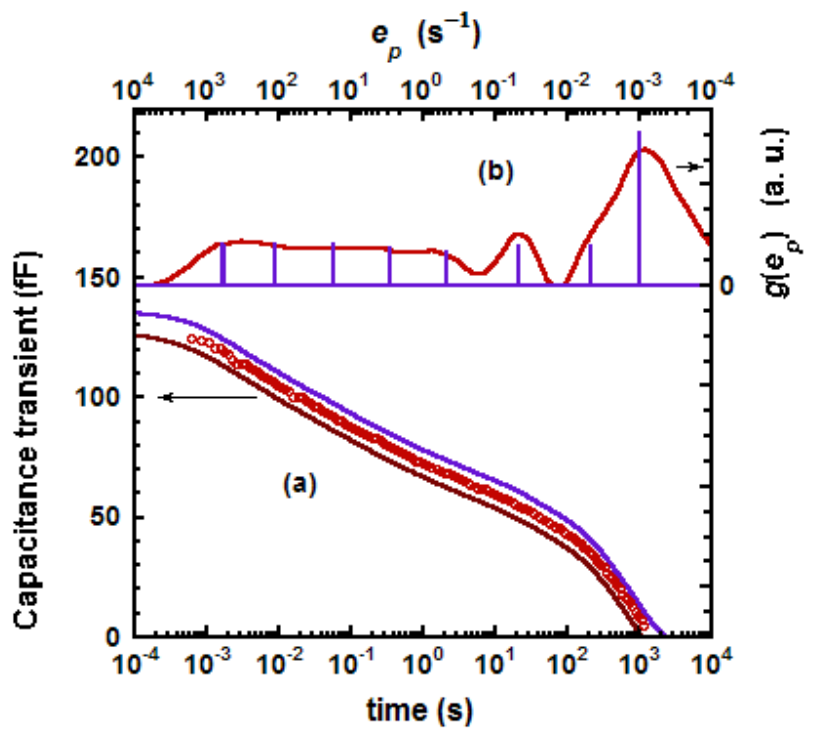

Figure 4 : (a) Experimental transient (open circles) measured at $500 \mathrm{~K}$ in the diode 3 of the sample A, which displays DLTS spectra (not shown) with intermediate FWMH between those of the diodes 1 and 2. (b) discrete and continuous distributions of emission rates deduced from the experimental transient (see text). In (a), solid lines are recalculated from these distributions, the upper from the discrete distribution, the lower from the continuous one, and shifted for clarity. 
Since the $e_{p}$ derivation is based on this law, such anomalies induce deviations from the straight line in the Arrhenius diagram, as displayed in Fig. 3 for the highest temperatures. More sophisticated methods become necessary in the case of multiple emission rates or continuous distribution. Equation (1) can be considered as still valid, because it comes from the detailed balance principle, but a sum of terms $\sum_{i} g_{i} \exp \left(-e_{p i} t\right)$ or an integral form must be

taken into account for the transient. The discrete distribution $g_{i}$ or the continuous one $g\left(e_{p}\right)$ has to be extracted by methods which both rely on time domain algorithms $[11,12]$ and measurements done in a very large time range, because of the dispersion of $e_{p i}$ or $e_{p}$ over several decades. Such a constraint requires transients recorded on a logarithmic time scale, typically over 6 decades, as shown in Fig. 4 (a). According to the algorithm which is used, either DISCRETE [11] or FTIKREG [12], the distribution which is deduced appears respectively as discrete or continuous in Fig. 4 (b). As shown in Fig. 4 (a), the transients simulated with either the discrete or the continuous distribution cannot be distinguished. The isothermal spectra would be useful for discriminating between the two models if both the experimental and simulated ones were available. Such a work, which needs large handling of data, is in progress. Meanwhile, from the extent of the emission rate distribution over approximately six decades in Fig. 4 (b), it is easy to derive a broadening of $6 \times 2.3 k T$ close to $0.6 \mathrm{eV}$ at the measurement temperature of $500 \mathrm{~K}$, if the dispersion is supposed to affect only the activation energy. Alternatively, a distribution of the capture cross sections over up to six decades could comply with the experimental results, but is less probable because capture cross section is strongly dependent of the initial charge of the centre, which is not expected to experience large fluctuations. For these reasons, the values deduced for the parameters $E_{a 1}$ and $\sigma_{p 1}$ must be considered with caution and at least as average values with larger uncertainties than initially quoted.

\subsection{Properties of the trap II}

A peak similar to that related to trap II in diode A is found for the sample B [9] in PICTS response and the values of $e_{p}(T)$ are aligned on the same straight line as for sample A in the Arrhenius diagram (see Fig. 3). This is the firm indication of a common origin of the trap II in both samples. Moreover, DLTS spectra of trap II display full width at half maximum (FWMH) very close to those of simulated ones in Fig. 1 and 2, indicating a single emission rate at each temperature. Then, the standard method used above for the derivation of $E_{a 2}$ and $\sigma_{p 2}$ can be considered as valid. Within the uncertainty previously quoted, a capture cross section in the $10^{-18} \mathrm{~cm}^{2}$ range suggests that the centre responsible for the trap II is repulsive for holes, and hence positively charged, involving a donor character. This prop- erty is confirmed by the following experiment performed in sample B.

In sample B, the PICTS signal has been measured when the sample was both heated and submitted to a temperature gradient from the back to its top surface, due to thermal losses. The subsequent inhomogeneous thermal expansion has triggered a plastic relaxation of the diamond layer, because of the additional stress. A strong increase of the dislocations density resulted as it can be seen in the cathodoluminescence spectra taken before and after thermal cycle (Fig. 5), since the A band due to dislocations at 2.9 $\mathrm{eV}$ increased by a factor of 40 . Moreover, the conductivity of the sample underwent a large decrease due to the compensation of boron acceptors, as already indicated in [9] and in a forthcoming publication. Compensation of acceptors must result necessarily from the presence of deep donors and therefore, trap II turns out to be a good candidate since it was detected after the decrease of the conductivity in sample B, displays a weak capture cross section in sample A and a common Arrhenius diagram in both samples. Moreover, the origin of this defect appears as connected to the presence of dislocations in the boron doped homoepitaxial layer.

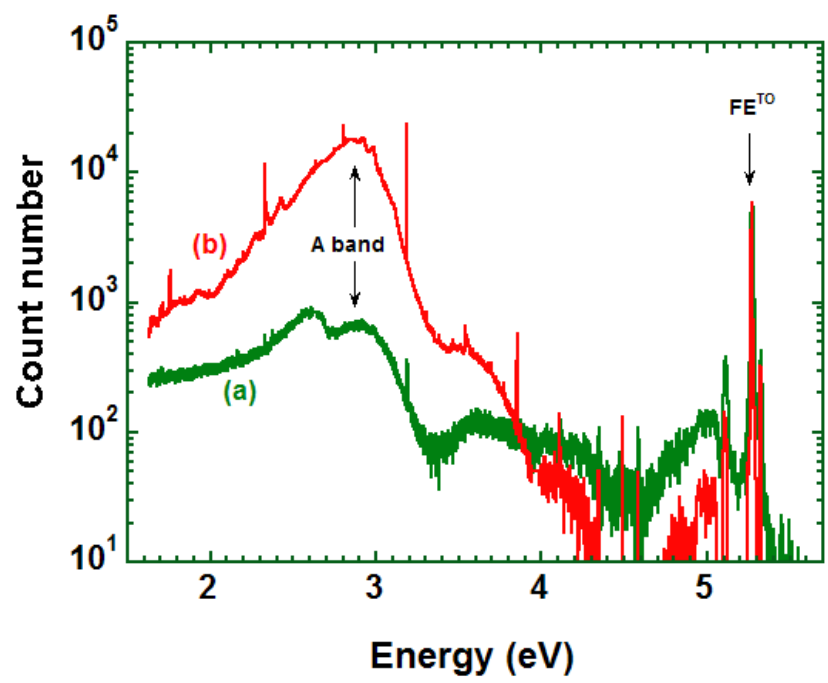

Figure 5 : Cathodoluminescence spectra taken at $5 \mathrm{~K}$ and normalised on the FETO line: (a) before heating from ambient to circa $650 \mathrm{~K}$; (b) after thermal cycle down to ambient temperature.

\section{Discussion and conclusions}

Study of defects in diamond has been performed essentially by optical methods [13], which probe radiative transition between a deep level and either the conduction band or valence band states, or even between two discrete levels like in donor-acceptor pair transitions. Hence, the final state is not necessarily known, contrary to the case of nonradiative transitions detected in DLTS. However, compari- 
son to data available in literature can be attempted. Several optical or photo-electrical studies [14-17] and one based on electrical measurements [18] show a transition close to 1.5 $\mathrm{eV}$ which could be compared to the case of trap I. But these results have all been obtained in samples where either impurities such as $\mathrm{Li}, \mathrm{Na}, \mathrm{S}$ or $\mathrm{N}$ have been deliberately introduced or the sample has been bombarded by ions. Neither the sample A nor B have undergone such treatments. Hence, one is forced to conclude that the origin of the trap I is not related to the impurities mentioned above. More generally, with a broad distribution of emission rates at each temperature as demonstrated in the second section, an impurity center is not likely. A complex defect, with several species and/or distorted neighbouring site, and also a fluctuating environment, is much more probable. Such variable structural changes near the defect site could be made easier with the presence of vacancies. It must also be noticed that some metastability of this defect has been detected since its concentration profile is both non monotonic and changes according to the subsequent thermal cycles [5]. As only hydrogen is able to move inside the diamond lattice, this set of conjectures suggest that this defect could be related to one of the complex involving a substitutional boron, a carbon vacancy and zero to four hydrogen atoms, as discussed by Goss et al. [19]. The deep donor, $1.13 \mathrm{eV}$ above the valence band edge, identified as the trap II, correlated with dislocations, may be tentatively ascribed to the positively charged vacancy, which is the stable state of the vacancy in boron doped diamond [20]. Detailed investigations, specially about the hydrogen content and profile will be helpful for a better understanding of these defects. The effect of non radiative transitions upon the deep levels population as probed by DLTS will remain the preferred method for studying this kind of problem, since it has already proven its interest in the present article.

\section{References}

[1] E. Kohn, A. Denisenko, Thin Solid Films 515, 4333 (2007).

[2] R. Kalish, J. Phys. D: Appl. Phys. 40, 6467 (2007).

[3] R. Zeisel, C. E. Nebel, M. Stutzmann, J. Appl. Phys. 84, 6105 (1998).

[4] P. Muret, C. Saby, F. Pruvost, A. Deneuville, Diamond Relat. Mater. 9, 1041 (2000).

[5] P. Muret, J. Pernot, T. Teraji, T. Ito, Appl. Phys. Express 1, 035003 (2008).

[6] T. Teraji, M. Hamada, H. Wada, M. Yamamoto, T. Ito, Diamond Relat. Mater. 14, 1747 (2005).

[7] T. Teraji, S. Mitani, T. Ito, phys. stat. sol. (a) 198, 395 (2003).

[8] M. Wade, P. Muret, F. Omnès, A. Deneuville, Diamond Relat. Mater. 15, 614 (2006).

[9] P. Muret, M. Wade, phys. stat. sol (a) 203, 3142 (2006)

[10] S. Weiss and R. Kassing: Solid-State Electron. 31, 1733 (1988).
[11] S. Provencher, Comput. Phys. Commun., 27, 213 (1982) and http://s-provencher.com/index.shtml

[12] J. Weese, Comput. Phys. Commun., 69, 99 (1992).

[13] A. M. Zaitsev, Optical Properties of Diamond (SpringerVerlag, Berlin, Heidelberg, 2001)

[14] M. Nesládek, K. Meykens, L.M. Stals, C. Quaeyhaegens, M. D'Olieslaeger, T.D. Wu, M. Vanecek, J. Rosa, Diamond Relat. Mater. 5, 1006 (1996)

[15] R. Zeisel, C. E. Nebel, M. Stutzmann, H. Sternschulte, M. Schreck, B. Stritzker, Phys. Stat. Sol. (a) 181, 45 (2000).

[16] J. A. Garrido, C. E. Nebel, and M. Stutzmann, E. Gheeraert, N. Casanova, and E. Bustarret, Phys. Rev. B 65, 165409 (2002).

[17] A. Hoffman, I. Andrienko, D.N. Jamieson, S. Prawer, Appl. Phys. Lett. 86, 044103 (2005).

[18] H. Sternschulte, M. Schreck, B. Stritzker, A. Bergmaier, G. Dollinger, Diamond Relat. Mater. 12, 318 (2003).

[19] J. P. Goss, P. R. Briddon, S. J. Sque, R. Jones, Phys. Rev. B 69, 165215 (2004).

[20] A. Mainwood, A. M. Stoneham, J. Phys.: Condens. Matter 9, 2453 (1997). 